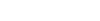

*For correspondence: john. broughan@ucd.ie

Competing interest: The authors declare that no competing interests exist.

Received: 04 February 2021 Accepted: 15 February 2021

Published: 12 May 2021

(T) This article is Open Access: CC BY license (https://creativecommons.org/licenses/by/4.0/)

Author Keywords: COVID-19, COVID-19 virus, SARS-CoV-2, follow-up studies, general practice, long-term care, pilot projects

Copyright (C) 2021, The Authors; DOI:10.3399/BJGPO.2021.0016

\title{
General practice attendances among patients attending a post-COVID-19 clinic: a pilot study
}

\begin{abstract}
John Broughan, BA(Hons), MSc ${ }^{1 *}$, Geoff McCombe, BSc, PhD1, Gordana Avramovic, BSc, MA ${ }^{1,2}$, Des Crowley, MSc, MB, MICGP, PhD1, Cheyenne Downey, BA, MSc ${ }^{1}$, Joanne Downey O'Sullivan, BSc, PGDip ${ }^{3}$, Ronan Fawsitt, MB, BCh, BAO, MRCGP, MICGP, DCH, DObs ${ }^{4,5}$, Tina McHugh, $\mathrm{MSc}^{1}$, Eileen O'Connor, BSc, H Dip, MSc${ }^{2}$, Carla Perrotta, MD, MPH, PhD', Aoife G Cotter, MB, PhD,7, John S Lambert, BA, MD, PhD ${ }^{1,2}$, Walter Cullen, MB, MD, MICGP, Grad Dip (T\&L), FFSEM (RCSI), FRCGP ${ }^{1}$
\end{abstract}

\begin{abstract}
Background: About 10-35\% of people with COVID-19 need medical care within 3 weeks of infection. However, the prevalence of ongoing care needs among those experiencing severe COVID-19 illness is unclear.
\end{abstract}

Aim: This pilot study aimed to address this knowledge gap by examining GP attendance trends among patients attending a post-COVID-19 hospital follow-up clinic, 3-6 months after an initial clinic visit.

Design \& setting: Data were collected from adult patients attending a post-COVID-19 follow-up clinic at the Mater Misericordiae University Hospital (MMUH), Dublin, Ireland.

Method: Participants completed questionnaires outlining their demographics; medical histories; emergency hospital admissions and readmissions where applicable; and, where relevant, GP attendances following hospital discharge. Analyses were conducted using descriptive and inferential statistics.

Results: Participants' $(n=153)$ median age was 43.5 years (interquartile range $[\mathrm{IQR}]=30.9-52.1$ years). There were 105 females (68.6\%, 95\% confidence interval $[\mathrm{Cl}]=61.3 \%$ to $75.9 \%$ ). Various medical histories were reported among participants. Sixty-seven $(43.2 \%, 95 \% \mathrm{Cl}=35.9 \%$ to $51.6 \%)$ received emergency COVID-19 hospital care. Older adults, males, intensive care unit [ICU] admissions, and readmissions were common among hospital attendees. Of the hospital attendees, $16(24 \%, 95 \%$ $\mathrm{Cl}=13.7 \%$ to $34.2 \%)$ attended GPs within 7 days of hospital discharge, and $26(39 \%, 95 \% \mathrm{Cl}=27.3 \%$ to $50.7 \%$ ) within 30 days. Older adults, people with pre-existing medical conditions, and individuals admitted to ICU and/or readmitted to hospital were common among general practice attendees.

Conclusion: Persistent health issues appear to be common among patients with severe COVID-19, particularly those who are older adults, have pre-existing health problems, and who had been in ICU and/or readmission care. Larger scale studies of ongoing COVID-19 care needs in primary care and general practice are required. 


\section{How this fits in}

This pilot study advances existing knowledge by highlighting the prevalence of the ongoing care needs of patients with severe COVID-19. The findings suggest that $39 \%(95 \% \mathrm{Cl}=27.3$ to $50.7 \%)$ of patients with severe COVID-19 require care within 30 days, the kind that is routinely provided by GPs. The findings also suggest that long-term care needs may be greater among older adults, patients with pre-existing health conditions, and patients requiring ICU and/or readmission care. Larger-scale studies in primary care and general practice are required to obtain more precise estimates of persistent illness among severe COVID-19 cases in communities.

\section{Introduction}

As of late January 2021, there have been 98925221 cases of COVID-19 globally, ${ }^{1}$ with 188923 cases in Ireland. ${ }^{2}$ Worldwide, 2127294 people have died with COVID-19 infection, ${ }^{1}$ and 2977 people have died with COVID-19 in Ireland. ${ }^{2}$ People of all ages can contract COVID-19, ${ }^{3}$ but those aged $<50$ years are more likely to do so. ${ }^{3}$ In contrast, individuals aged $>50$ years are more likely to die because of COVID-19 infection. ${ }^{3}$ Both males and females have similar chances of being infected with COVID-19, 4,5 and in Ireland, COVID-19 death rates are similar for both sexes. ${ }^{5}$ However, international research shows that males are more likely to experience severe clinical outcomes, hospitalisation, ICU admittance, and mortality. ${ }^{4,6,7}$

Immunocompromised populations (for example, people receiving cancer therapies such as chemotherapy, radiotherapy, and immunotherapy; and individuals with HIV) appear more likely to contract COVID-19. ${ }^{8,9}$ Comorbidities such as hypertension, diabetes mellitus, cardiovascular diseases, and chronic obstructive pulmonary diseases are also common among those with COVID-19. ${ }^{10}$ To compound matters, individuals with pre-existing health conditions are often more susceptible to severe COVID-19-related illness and injury. At-risk populations include those with weaker immune systems (for example, recipients of immunosuppressant therapy and populations with HIV), ${ }^{8,9}$ and those with chronic health conditions such as hypertension, ${ }^{10,11}$ diabetes, ${ }^{10,11}$ cardiac illnesses, ${ }^{4,10-12}$ chronic lung disease, ${ }^{13}$ liver disease, ${ }^{14}$ haematological malignancy, ${ }^{15}$ chronic kidney disease, ${ }^{16}$ and neurological conditions including dementia, Parkinson's, epilepsy, multiple sclerosis, and spinal cord injury. ${ }^{16}$ Evidence regarding COVID-19-related health impacts for groups — including pregnant patients and their babies, ${ }^{17}$ patients who are immunocompromised, patients with diabetes, ${ }^{9,18}$ and patients with asthma ${ }^{19}$ - is mixed in terms of clinical outcomes and evidence availability.

Research concerning COVID-19 hospitalisation rates, experiences, and longer-term follow up is varied. Data from the UK and Ireland shows that hospitalisation rates have varied both between countries and as the pandemic has progressed over time. ${ }^{20,21}$ Length of hospital stay, meanwhile, was estimated as 4-53 days in China, with shorter stays (4-21 days) reported elsewhere. ${ }^{22}$ Such discrepancies have been attributed to country-specific differences in admission and discharge criteria. ${ }^{22}$ Reported COVID-19 ICU rates have also varied. In Ireland, ICU rates are estimated to be between $1 \%$ and $2 \%,{ }^{5}$ while a systematic review of international literature calculated a pooled ICU rate of $15.4 \%$, with $14.9 \%$ of patients requiring mechanical ventilation. ${ }^{23}$ Research also suggests that COVID-19 hospital readmission rates are low, ${ }^{24}$ with COVID-19 readmissions most likely to occur among immunocompromised and co-morbid populations. ${ }^{24,25}$ Early rather than later readmissions are more associated with COVID-19 related illness, as opposed to illness arising from pre-existing health conditions. ${ }^{25}$

In the UK, approximately $10 \%$ of those diagnosed with COVID-19 require, but do not necessarily receive, care within 3 weeks of infection. ${ }^{26}$ In contrast, research in the US has shown that 35\% of people with COVID-19 feel unwell 2-3 weeks following positive diagnosis. ${ }^{27}$ Either way, it is evident that many people recovering from COVID-19 require ongoing care, the kind that is routinely provided in general practice. ${ }^{26,28}$ However, the extent to which those recovering from severe COVID-19 have attended general practice is uncertain. This pilot study sought to address this knowledge gap by investigating the recent GP service use trends of patients with COVID-19 attending a post-COVID-19 follow-up clinic at the Mater Misericordiae University Hospital (MMUH) in Dublin, Ireland. 


\section{Method}

This study was conducted and reported according to the STROBE (Strengthening the Reporting of Observational Studies in Epidemiology) guidelines. ${ }^{29}$ The study protocol on which it is based has been reported separately. ${ }^{30}$ This study's methods deviate somewhat from those outlined in the protocol. For instance, unlike in the protocol, data collection occurred at the MMUH's post-COVID-19 clinic rather than at general practices in North Dublin. Further, this study had a smaller sample size than was outlined in the protocol, and this study did not examine patients' COVID-19 clinical outcomes.

\section{Setting}

The study was conducted at the post-COVID-19 follow-up clinic at the MMUH Dublin, Ireland. It is a once-weekly outpatient clinic allowing patients recovering from COVID-19 to be followed up and assessed for persistent COVID-19 symptoms. The patients consist of individuals who have: (a) been hospitalised with COVID-19; (b) been placed on a COVID-19 ambulatory home monitoring programme; and/or (c) been referred from local GPs with COVID-19 infection. Clinical assessment is conducted at the clinic, and relevant diagnostic tests are ordered as clinically indicated (for example, pathology tests, chest Xrays, echocardiography, pulmonary function tests, or computed tomography [CT] of thorax). Patients with persistent symptoms are subsequently referred to a specialist for assessment and treatment.

\section{Participants}

Participants were adult patients seen at the clinic between June and November 2020, and who provided informed consent to participate in the study. Approximately 12-15 patients were offered appointments at the clinic each week. Thus, it is estimated that 250-300 patients were invited to attend the clinic during the entire study period.

\section{Procedure}

Consenting participants completed questionnaires administered by various researcher or healthcare professionals, querying participants' demographic profiles; prior medical histories; COVID-19 infection details; emergency hospital admissions and readmissions, where applicable; and, where relevant, GP service use trends following hospital discharge.

\section{Instruments}

Participants were asked to indicate their age, sex, and whether they have experienced any of the following conditions: pregnancy, cancer, diabetes, HIV (or other immune-deficiency diseases), heart disease, asthma (requiring medication), other chronic lung diseases, chronic liver disease, haematological disorder, kidney disease, neurological impairment or disease, organ or bone marrow transplant, or 'other pre-existing condition(s)'. Participants were asked about COVID-19-related hospital experiences, with questions focusing on whether participants were hospitalised, admitted to ICU, and/or readmitted to hospital following an initial stay. Participants were also asked to outline the duration(s) of the above experiences, if relevant. Lastly, where applicable, participants were asked to document whether they had attended GP services within 7 and 30 days of their COVID-19-related hospital discharge. 


\section{Analysis}

Data were analysed using descriptive and inferential statistical methods via SPSS (version 26).

\section{Results}

The sample's $(n=153$ ) median age was 43.5 years ( $I Q R=30.9-52.1$ years). There were 105 females (68.6\%, $95 \% \mathrm{Cl}=61.3 \%$ to $75.9 \%)$. Various medical histories were reported among participants, the prevalence of which is outlined below (see Table 1). Sixty-seven participants $(43.8 \%, 95 \% \mathrm{Cl}=35.9 \%$ to $51.6 \%$ ) reported being admitted to hospital with COVID-19. Data outlining the proportion and characteristics of ambulatory home monitoring programme patients, and patients referred to the clinic by GPs were not available.

\section{Hospital experiences}

Participants' median hospital stay was 7 days (IQR $=2-15$ days). Older individuals were more likely to be hospitalised, $P \leq 0.001(95 \% \mathrm{Cl}=8.9$ to 16.8$)$, as were males, $P \leq 0.001 ;(\phi=-0.283)$, and people with pre-existing health conditions, $P \leq 0.001$; $(\phi=0.338)$, particularly those with heart disease, $P=$ $0.005,(\phi=0.246)$. Nine participants $(5.9 \%, 95 \% \mathrm{Cl}=0 \%$ to $11.5 \%)$ were admitted to ICU, with median ICU stays of 25 days (IQR $=17-37.5$ days). Hospitalised patients with chronic lung disease were likely to require ICU care, $P=0.045$, $(\phi=0.338)$. Seventeen participants $(11.1 \%, 95 \% \mathrm{Cl}=3.5 \%$ to $18.6 \%)$ had been readmitted to hospital since an initial COVID-19 hospital stay. These patients had a median readmission stay of 3 days (IQR $=1-5.8$ days).

\section{Patients' GP attendances}

Of the 67 participants admitted to hospital, $16(24 \%, 95 \% \mathrm{Cl}=13.7 \%$ to $34.2 \%)$ reported attending a GP within 7 days of hospital discharge, and $26(39 \%, 95 \% \mathrm{Cl}=27.3 \%$ to $50.7 \%)$ reported attending a GP within 30 days of discharge. Data concerning recent GP service usage among the ambulatory home monitoring programme patients, and patients referred to the clinic by GPs were not available.

\section{Within 7 days of hospital discharge}

The 16 participants attending GPs within 7 days of hospital discharge had a median age of 50.3 years $(\mathrm{IQR}=41.7-58.4$ years). Eight were male $(50 \%, 95 \% \mathrm{Cl}=25.5 \%$ to $74.5 \%)$, three had cancer $(18.8 \%$, $95 \% \mathrm{Cl}=0 \%$ to $37.9 \%)$, three had diabetes $(18.8 \%, 95 \% \mathrm{Cl}=0 \%$ to $37.9 \%)$, three had heart disease $(18.8 \%, 95 \% \mathrm{Cl}=0 \%$ to $37.9 \%)$, one had chronic lung disease $(6.3 \%, 95 \% \mathrm{Cl}=0 \%$ to $18.2 \%)$, one had a chronic neurological impairment or disease $(6.3 \%, 95 \% \mathrm{Cl}=0 \%$ to $18.2 \%)$, and $15(93.8 \%, 95 \%$ $\mathrm{Cl}=81.9 \%$ to $100 \%$ ) had 'other pre-existing condition(s)'. Participants' median hospital stay was 6.5 days (IQR $=3-14.3$ days). Three were admitted to ICU and their ICU stays were 14, 25, and 26 days, respectively. Seven participants $(43.8 \%, 95 \% \mathrm{Cl}=19.4 \%$ to $68.1 \%)$ were readmitted to hospital since their initial visit. All readmission stays lasted less than 1 week.

\section{Within 30 days of hospital discharge}

Those attending GPs within 30 days of hospital discharge had a median age of 50.4 years (IQR $=44$, $1-57.1$ years). This group consisted of 26 patients, of which 13 were males $(50 \%, 95 \% \mathrm{Cl}=30.7 \%$ to $69.2 \%)$. Five participants $(19.2 \%, 95 \% \mathrm{Cl}=4 \%$ to $34.3 \%)$ had diabetes, five $(19.2 \%, 95 \% \mathrm{Cl}=4 \%$ to $34.3 \%)$ had heart disease, three $(11.5 \%, 95 \% \mathrm{Cl}=0 \%$ to $23.7 \%)$ had cancer, three $(11.5 \%, 95 \% \mathrm{Cl}=$ $0 \%$ to $23.7 \%$ ) had chronic lung disease, one $(3.8 \%, 95 \% \mathrm{Cl}=3.5 \%$ to $11.1 \%)$ had asthma requiring medication, one $(3.8 \%, 95 \% \mathrm{Cl}=3.5 \%$ to $11.1 \%)$ had a chronic neurological impairment or disease, and $23(88.5 \%, 95 \% \mathrm{Cl}=76.2 \%$ to $100 \%)$ had an 'other pre-existing condition'. The median hospital stay for this group was 6 days (IQR $=2.8-15$ days). Five participants were admitted to ICU. The median ICU stay was 26 days (IQR $=19.5-58.5$ days). Ten participants $(38.5 \%, 95 \% \mathrm{Cl}=19.8 \%$ to $57.2 \%$ ) had been readmitted to hospital since their last COVID-19-related visit, with all readmission stays lasting 3 days or less. When compared to all other responding participants in the sample (that is, both hospitalised and non-hospitalised participants, $n=153$ ), those attending GPs within 30 days of hospital discharge were: (a) significantly older, $P=0.011(95 \% \mathrm{Cl}=1.6$ to 11.8$)$; (b) more likely to have chronic lung disease, $P=0.015(\phi=0.252)$; and (c) more likely to have 'other pre-existing health conditions', $P=0.010(\phi=0.215)$. 


\section{Discussion}

\section{Summary}

The findings showed that $24 \%$ and $39 \%$ of patients hospitalised with COVID-19 attended general practice within 7 and 30 days following hospital discharge, respectively. While the findings cannot demonstrate a causal relationship between participants' GP consultations and COVID-19 health complications requiring follow-up, it is believed it is likely that many of their consultations were COVID-19 related. The authors believe this because the study's GP attendees possessed characteristics previously linked to persistent COVID-19 complications. For instance, the GP attendees in the sample had an average age of approximately 50 years, several had pre-existing medical conditions, and several others had been admitted to ICU, and/or were readmitted to hospital with further COVID-19related health complications.

\section{Strengths and limitations}

The COVID-19 clinic at the MMUH, Dublin, and the clinical staff working there, played an instrumental role with regards to recruiting and collecting data from this study's participants. The clinic facilitated routine access to a hard-to-reach population during a period of strict COVID-19 restrictions on movement in Ireland, and so its contribution to this study cannot be underestimated. Further, the study's reporting process benefited from adopting the STROBE guidelines for observational studies, ${ }^{29}$ and from guidelines outlined in the study protocol. ${ }^{30}$ The generalisability of this study's findings, meanwhile, are limited by the smaller size of the sample, particularly the GP attendee samples, and by the fact that convenience rather than random sampling techniques were used for feasibility purposes. Also, participants were not questioned as to whether their GP visits were COVID-19 related. Thus, the findings could not demonstrate a causal relationship between COVID-19 illness and general practice attendances. Lastly, the authors did not have data outlining the recent GP service use trends of patients in the sample who were not hospitalised (that is, those placed on the COVID-19 ambulatory home monitoring programme, and those referred to the clinic from local GPs). It is unclear whether these groups' GP service usage trends would have differed from those of the hospitalised group.

\section{Comparison with existing literature}

The finding that $24 \%$ and $39 \%$ of patients hospitalised with COVID-19 require follow-up care contrasts with estimates for all individuals diagnosed COVID-19 with COVID-19 from the UK (10\%) ${ }^{26}$ and the US (35\%). ${ }^{27}$ The findings also provide support for claims that older adults ${ }^{3}$ and individuals with preexisting health conditions ${ }^{9-13}$ are more likely to experience ongoing COVID-19 complications requiring follow-up. Moreover, it was notable that this study's GP attendees had higher readmission rates than those observed among the general population in previous studies. ${ }^{24,25}$

\section{Implications for research and practice}

This pilot study's findings suggest that persistent COVID-19 complications could be a serious public health concern. Thus, larger-scale studies are necessary to obtain more precise estimates of persistent illness following severe COVID-19 cases in the general population(s). It is recommended that such studies consult the Method and Strengths and limitations sections of this article for guidance on how to design their research. Qualitative research and studies comparing the prevalence of persistent COVID-19 illness between countries with varying socioeconomic profiles, health indexes, COVID-19 response strategies, and healthcare systems may also be informative. Meanwhile, the authors acknowledge that this was a pilot study with a small sample, and that any recommendations that are made for clinical practice must be done cautiously. With this in mind, the authors simply ask that clinicians be mindful of persistent COVID-19 health issues in the community, vulnerable groups that may be more susceptible to persistent COVID-19 concerns, and relevant ongoing research activities and findings.

In conclusion, this pilot study's findings suggest that persistent health concerns requiring ongoing care may be common among people with severe COVID-19 infection. The findings also suggest that among populations with severe COVID-19, older adults, individuals with pre-existing medical conditions, and those requiring ICU and/or hospital readmission may have greater ongoing care 
needs. Large scale studies are required to gain more precise estimates of persistent illness among severe COVID-19 cases in the general population(s).

\section{Funding}

This study is funded by the Health Research Board (reference number: COV19-2020-123). The study contributes to a wider body of work being produced to attenuate the adverse effects of the COVID-19 pandemic on population health in Ireland (The North-Dublin COVID-19 Cohort ['ANTICIPATE'] Study). The study also received support from the Ireland East Hospital Group, and internal funding schemes at the UCD School of Medicine, and the UCD College of Health and Agricultural Sciences.

\section{Ethical approval}

The study was approved by the Mater Misericordiae University Hospital Research Ethics Committee (reference number: 1/378/2141).

\section{Provenance}

Freely submitted; externally peer reviewed.

\section{Acknowledgements}

The authors would like to thank Ireland's Health Research Board (HRB) [COV19-2020-123] and the Ireland East Hospital Group, as well as internal funding schemes at the UCD School of Medicine, and the UCD College of Health and Agricultural Sciences for supporting this project [COV19-2020-123]. They would like to acknowledge the efforts of clinical staff at the MMUH's post-COVID-19 follow-up clinic. They would also like to thank all the study's participants, as well as the following personnel for their ongoing contributions to these COVID-19 research efforts: Mary-Carmel Burke, Allys Guerandel, Eilis Hennessy, Cecily Kelleher, and Gerald Mills.

\section{References}

1. World Health Organization. WHO coronavirus (COVID-19) dashboard. 2020; https://covid19.who.int/ (accessed 9 Apr 2021).

2. Government of Ireland. Ireland's COVID-19 Data Hub. 2020; https://covid-19.geohive.ie/ (accessed 9 Apr 2021).

3. Carethers JM. Insights into disparities observed with COVID-19. J Intern Med 2021; 289(4): 463-473. DOI: https:// doi.org/10.1111/joim.13199

4. Gebhard C, Regitz-Zagrosek V, Neuhauser HK, et al. Impact of sex and gender on COVID-19 outcomes in Europe. Biol Sex Differ 2020; 11(1): 29. DOI: https://doi.org/10.1186/s13293-020-00304-9

5. Health Promotion Surveillance Centre (HPSC). Epidemiology of COVID-19 in Ireland. Dublin: HPSC; 2020.

6. Lakbar I, Luque-Paz D, Mege J-L, et al. COVID-19 gender susceptibility and outcomes: a systematic review. PLoS One 2020; 15(11): e0241827. DOI: https://doi.org/10.1371/journal.pone.0241827

7. Mohamed MS, Moulin TC, Schiöth HB. Sex differences in COVID-19: the role of androgens in disease severity and progression. Endocrine 2021; 71(1): 3-8. DOI: https://doi.org/10.1007/s12020-020-02536-6

8. Morani Z, Patel S, Ghosh S, et al. COVID-19 in HIV: a review of published case reports. SN Compr Clin Med 2020; 2: 2647-2657. DOI: https://doi.org/10.1007/s42399-020-00593-6

9. Seth G, Sethi S, Bhattarai S, et al. SARS-CoV-2 infection in cancer patients: effects on disease outcomes and patient prognosis. Cancers 2020; 12(11): 3266. DOI: https://doi.org/10.3390/cancers12113266

10. Tadic M, Cuspidi C, Sala C. COVID-19 and diabetes: is there enough evidence? J Clin Hypertens 2020; 22(6): 943-948. DOI: https://doi.org/10.1111/jch.13912

11. Radwan NM, Mahmoud NE, Alfaifi AH, Alabdulkareem KI. Comorbidities and severity of coronavirus disease 2019 patients. Saudi Med J 2020; 41(11): 1165-1174. DOI: https://doi.org/10.15537/smj.2020.11.25454

12. Alsaied T, Aboulhosn JA, Cotts TB, et al. Coronavirus disease 2019 (COVID-19) pandemic implications in pediatric and adult congenital heart disease. J Am Heart Assoc 2020; 9(12): e017224. DOI: https://doi.org/10.1161/JAHA. 120.017224

13. Çakır Edis E. Chronic pulmonary diseases and COVID-19. Turk Thorac J 2020; 21(5): 345-349. DOI: https://doi.org/ 10.5152/TurkThoracJ.2020.20091

14. Sharma A, Jaiswal P, Kerakhan Y, et al. Liver disease and outcomes among COVID-19 hospitalized patients - A systematic review and meta-analysis. Ann Hepatol 2021; 21: 100273. DOI: https://doi.org/10.1016/j.aohep.2020. 10.001

15. El-Sharkawi D, lyengar S. Haematological cancers and the risk of severe COVID-19: exploration and critical evaluation of the evidence to date. Br J Haematol 2020; 190(3): 336-345. DOI: https://doi.org/10.1111/bjh.16956

16. Kubota T, Kuroda N. Exacerbation of neurological symptoms and COVID-19 severity in patients with preexisting neurological disorders and COVID-19: a systematic review. Clin Neurol Neurosurg 2021; 200: 106349. DOI: https:// doi.org/10.1016/j.clineuro.2020.106349 
17. Di Toro F, Gjoka M, Di Lorenzo G, et al. Impact of COVID-19 on maternal and neonatal outcomes: a systematic review and meta-analysis. Clin Microbiol Infect 2021; 27(1): 36-46. DOI: https://doi.org/10.1016/j.cmi.2020.10.007

18. Corse T, Dayan L, Kersten S, et al. Clinical outcomes of COVID-19 patients with pre-existing, compromised immune systems: a review of case reports. Int J Med Sci 2020; 17(18): 2974-2986. DOI: https://doi.org/10.7150/ ijms. 50537

19. Hughes-Visentin A, Paul ABM. Asthma and COVID-19: what do we know now. Clin Med Insights Circ Respir Pulm Med 2020; 14: 117954842096624. DOI: https://doi.org/10.1177/1179548420966242

20. Cunniffe NG, Gunter SJ, Brown M, et al. How achievable are COVID-19 clinical trial recruitment targets? A UK observational cohort study and trials registry analysis. BMJ Open 2020; 10(10): e044566. DOI: https://doi.org/10. 1136/bmjopen-2020-044566

21. Kennelly B, O'Callaghan M, Coughlan D, et al. The COVID-19 pandemic in Ireland: an overview of the health service and economic policy response. Health Policy Technol 2020; 9(4): 419-429. DOI: https://doi.org/10.1016/j.hlpt. 2020.08.021

22. Demeco A, Marotta N, Barletta M, et al. Rehabilitation of patients post-COVID-19 infection: a literature review. J Int Med Res 2020; 48(8): 030006052094838. DOl: https://doi.org/10.1177/0300060520948382

23. Patel U, Malik P, Mehta D, et al. Early epidemiological indicators, outcomes, and interventions of COVID-19 pandemic: a systematic review. J Glob Health 2020; 10(2): 020506. DOI: https://doi.org/10.7189/jogh.10.020506

24. Parra LM, Cantero M, Morrás I, et al. Hospital readmissions of discharged patients with COVID-19. Int J Gen Med 2020; 13: 1359-1366. DOI: https://doi.org/10.2147/IJGM.S275775

25. Atalla $\mathrm{E}$, Kalligeros M, Giampaolo G, et al. Readmissions among patients with COVID-19. Int J Clin Pract; 75: e13700. DOI: https://doi.org/10.1111/ijcp.13700

26. Greenhalgh T, Knight M, A'Court C, et al. Management of post-acute covid-19 in primary care. BMJ 2020; 370: m3026. DOI: https://doi.org/10.1136/bmj.m3026

27. Tenforde MW, Kim SS, Lindsell CJ, et al. Symptom duration and risk factors for delayed return to usual health among outpatients with COVID-19 in a multistate health care systems network - United States, March-June 2020. MMWR Morb Mortal Wkly Rep 2020; 69(30): 993-998. DOI: https://doi.org/10.15585/mmwr.mm6930e1

28. Kumar R, Nedungalaparambil NM, Mohanan N. Emergency and primary care collaboration during COVID-19 pandemic: a quick systematic review of reviews. J Family Med Prim Care 2020; 9(8): 3856-3862. DOI: https://doi. org/10.4103/jfmpc.jfmpc_755_20

29. Poorolajal J, Cheraghi Z, Irani AD, Rezaeian S. Quality of cohort studies reporting post the strengthening the reporting of observational studies in epidemiology (STROBE) statement. Epidemiol Health 2011; 33 : e2011005. DOI: https://doi.org/10.4178/epih/e2011005

30. Cullen W, Avramovic G, Broughan J, et al. Study protocol: prospective, observational, cohort study of COVID-19 in general practice (North Dublin COVID-19 cohort ['ANTICIPATE'] study [version 1; peer review: 2 approved with reservations]. HRB Open Res 2020; 3: 67. DOI: https://doi.org/10.12688/hrbopenres.13135.1 